\title{
Reuna
}

\section{CUSTO TOTAL PARA O CONSUMIDOR E CUSTEIO DO CICLO DE VIDA: REFLEXÕES, FINALIDADES E COMPARAÇÕES}

\section{TOTAL COST OF OWNERSHIP AND LIFE CYCLE COST: REFLECTIONS, PURPOSES AND COMPARISONS}

\author{
http://dx.doi.org/10.21714/2179-8834/2018v23n1p75-96
}

\author{
Paulo Henrique Rezende da Silva \\ Universidade Estadual de Maringá (UEM), Brasil. \\ E-mail: pauloh_r_s@hotmail.com \\ Lucas Merotti Barbosa \\ Universidade Estadual de Maringá (UEM), Brasil. \\ E-mail: merottibarbosa@hotmail.com \\ Katia Abbas \\ Universidade Estadual de Maringá (UEM), Brasil. \\ E-mail: katia_abbas@yahoo.com.br \\ Kelly Cristina Mucio Marques \\ Universidade Estadual de Maringá (UEM), Brasil. \\ E-mail: kcmmarques@uem.br \\ Juliane Andressa Pavão \\ Universidade Estadual de Maringá (UEM), Brasil. \\ E-mail: julianepavao@hotmail.com
}

Submissão: 01 Junho 2017 Publicação: 09 Abril 2018. Sistema de avaliação: Double blind review. Centro Universitário UNA, Belo Horizonte - MG, Brasil. Editor geral: Prof. Dr. Gustavo Quiroga Souki

Este artigo encontra-se disponível nos seguintes endereços eletrônicos:

http://revistas.una.br/index.php/reuna/article/view/934

http://dx.doi.org/10.21714/2179-8834/2018v23n1p75-96

\section{RESUMO}

O objetivo deste estudo é identificar e analisar as diferenças e semelhanças entre o Custo Total para o Consumidor (CTC) e o Custeio do Ciclo de Vida (CCV), tendo em vista a percepção de falta de clareza e de concordância entre os autores e a consequente não difusão na prática ao mesmo nível dos demais artefatos da Gestão Estratégica de Custos. Foram necessários levantamentos na literatura nacional e internacional sobre os temas, a fim de possibilitar a análise das diferentes definições e abordagens, bem como a busca por uma distinção clara entre os dois artefatos estratégicos. O estudo se caracteriza como uma análise crítico-interpretativa da literatura. Percebe-se que existe realmente certa confusão frente às diferentes definições, principalmente, por caminharem rumo a um mesmo ponto de convergência estratégico. Alguns conceituam como sendo iguais, outros consideram que o CTC está incluso no CCV, ou ainda o contrário. A partir das diferentes visões e reflexões apresentadas coube construir uma representação gráfica, visando contribuir com a diferenciação entre os artefatos. Por fim, foi apresentado um quadro contendo as características essenciais identificadas. Foi constatada, na maioria dos autores, uma 
visão do CCV mais abrangente, considerando custos desde a pesquisa e desenvolvimento até o descarte do produto, enquanto o CTC parte da aquisição do produto, ou seja, o momento da escolha e compra, além dos possíveis gastos provenientes do consumo até o descarte.

Palavras-chave: Gestão Estratégica de Custos; Ciclo de Vida; Custo Total para o Consumidor.

\section{ABSTRACT}

The objective of this study is to identify and analyze the differences and similarities between the Total Cost of Ownership (TCO) and the Life-Cycle Cost (LCC) in order to perceive a lack of clarity and agreement between the authors and the consequent not diffusion in practice to the same level of the other artifacts of the Strategic Cost Management. It was necessary to draw up national and international literature on the themes in order to allow the analysis of the different definitions and approaches, as well as the search for a clear distinction between the two strategic artifacts. The study is characterized as a critical-interpretive analysis of the literature. It is perceived that there is indeed some confusion over the different definitions, especially as they move towards the same point of strategic convergence. Some conceptualize as being equal, others consider that the TCO is included in the LCC or the opposite. From the different views and reflections presented, it was possible to construct a graphic representation in order to contribute to the differentiation between the artifacts. Finally, a table containing the essential characteristics identified was presented. In most of the authors, a more comprehensive view of the LCC was considered, considering costs from research and development to product disposal, while the TCO starts from the product acquisition, that is, the moment of choice and purchase, besides the possible from consumption to disposal.

Keywords: Strategic Cost Management; Life-Cycle Cost; Total Cost of Ownership.

\section{Introdução}

Já é praxe no início de textos científicos citar a globalização, evolução de mercados e avanços na competição empresarial como terreno para delinear qualquer tipo de fenômeno. De fato, essas são características irrefutáveis ao avanço da economia e também das práticas contábeis. Não só esses, mas também a atenção à qualidade, melhoria nas políticas de gestão, estrutura industrial de alto nível e a busca incessante na redução de custos, segundo Nakagawa (1993), são fatores que não podem deixar de ser citados.

Saccani, Perona e Bacchetti (2017) e Mendes, Barbosa Neto e Dias (2012) ressaltam a dinamicidade do cenário competitivo atual, munido de disputas por demandas, aumento constante de concorrência e de estratégias de preços definidas e analisadas amplamente, inclusive com participação dos fornecedores e demais envolvidos, tudo com o objetivo de buscar cada vez mais novos mercados, disputa essa que exige informações ágeis sobre custos e desempenho, em perspectiva de longo prazo e em caráter flexível, sempre adepta a mudanças (MENDES, BARBOSA NETO; DIAS, 2012). 
Dado o contexto evolutivo e dinâmico, a contabilidade de custos avançou, gradativamente, nos últimos trinta anos, proporcionando além das ferramentas para mensuração dos custos dos produtos, controle e análises gerenciais, ferramentas focadas na visão estratégica. Esse novo desdobramento, divulgado mais intensamente com as obras de Porter, publicadas entre 1979 e 1991, recebeu o nome de Gestão Estratégica de Custos. Além de Porter, outros autores que merecem destaque são Shank e Govindarajan, com a obra publicada em 1993 e intitulada: "Strategic Cost Management: the new tool for competitive advantage", que tem recebido contínuas citações tanto em estudos nacionais quanto internacionais.

O diferencial em relação à Contabilidade de Custos tradicional se deve a análises sob um ambiente mais amplo, no qual os elementos estratégicos são mais claros e formados a fim de obter a vantagem competitiva sustentável. Nesse contexto, Shank e Govindarajan (1997) acrescentam que as informações de custos são utilizadas para a construção de estratégias, porém deve haver nos gestores e contadores gerenciais uma mudança de forma de pensar ou no que acreditar, ou seja, deve-se olhar de um jeito novo uma base de dados gerenciais de custos já disponíveis em modo tradicional.

A nova vertente oferecida por meio da Gestão Estratégica de Custos (GEC), portanto, é a resposta à demanda do contexto organizacional por formas mais precisas, coesas e estratégicas de atuar perante o mercado e os concorrentes. A combinação de Contabilidade de Custos e Estratégia reporta a uma nova ótica e à origem de artefatos, que objetivam suprir essas necessidades.

Assim, afloram vários artefatos da GEC, cada qual com características conceituais e procedimentais similares, mas que mantêm suas diferenças e caminham rumo a um mesmo objetivo: a busca por vantagem competitiva. Alguns desses fragmentos, porém, por serem relativamente recentes, não possuem frameworks munidos de unicidade e clareza de definições, o que pode resultar em possíveis confusões ou faltas de entendimento e de aplicação na prática (LANGFIELD-SMITH, 2008). Slavov (2013) compartilha da mesma opinião, afirmando que alguns artefatos estratégicos são recentes e pouco estruturados, remetendo à falta de um corpo unificado capaz de estabelecer princípios claros e precisos, o que acaba por resultar em confusões nas definições usadas nas pesquisas e na prática, devido à falta de fundamentação e consistência de definições. Paira sobre alguns artefatos gerenciais a diversidade de definições, que possivelmente ocasionam confusões conceituais, haja vista que alguns guardam grandes semelhanças (MIAH, KOH; STONE, 2017), contexto esse que pode ser percebido no caso do custeio do ciclo de vida (CCV) e do custo total para o consumidor (CTC) ou Total Cost of Ownership (TCO), no inglês, focos deste estudo.

O CCV "lida explicitamente com a relação entre o que o consumidor paga por um produto e o custo total em que o consumidor incorre no ciclo de vida útil do produto" (SHANK; GOVINDARAJAN, 1997, p. 16). O objetivo é mensurar, analisar e gerenciar os custos incorridos em cada etapa do ciclo de vida, ou seja, na concepção, no desenvolvimento, na produção, no uso e no descarte. Nakagawa (1993) cita que a contribuição está no suporte à empresa com foco na otimização de desempenho, o que torna evidente todos os fatores que influenciam o desempenho ao decorrer do tempo. 
Já o CTC, segundo Ellram e Siferd (1998), é uma abordagem complexa em que o consumidor, ao optar pela compra de um produto, deve considerar não apenas o preço que será pago, mas também outros itens relevantes envolvidos na aquisição, posse, uso e subsequente destruição. Assim, são considerados os custos com ordem de colocação, de pesquisa e de qualificação de fornecedores, de transporte, de recebimento, de inspeção, de rejeição, de armazenamento e de eliminação. Em meio a um mercado altamente competitivo, com saturação de demanda e constante aumento de concorrência, conhecendo bem o consumidor e utilizando uma abordagem em longo prazo e orientada para o mercado, a empresa acaba auferindo maiores possibilidades de reduzir seu custo total de propriedade perante a cadeia de valor, ao mesmo tempo em que beneficia e conquista o cliente por meio de suas decisões de compra (SACCANI, PERONA; BACCHETTI, 2017).

Devido ao fato das definições desses dois artefatos da GEC manterem grande proximidade, a literatura pode apresentar ausência de clareza quanto às definições e finalidades e, apesar de apresentarem semelhanças, mantêm suas peculiaridades, argumento firmado por Ferrin e Plank (2002), quando afirmam que há um relacionamento próximo entre os dois artefatos, que desencadeia várias conexões e complementos entre um e outro. Um exemplo de tal argumento se apresenta no estudo de Dias Filho, Nakagawa e Rocha (2002), que almejam explorar a utilidade do CCV, mas abordam, na maioria do conteúdo do tópico referente, definições relacionadas ao CTC, como no trecho que mostram a forma como o CCV pode representar uma ferramenta de vantagem competitiva, sendo que na sequência das ideias é ressaltado que esse suporte pode ser oferecido, realmente, por meio de definições do custo total. Outro estudo em que é perceptível essa conexão entre CTC e CCV é em Okano (2001), em que se afirma que o CCV é um processo de análise econômica e uma técnica de avaliação para cálculo e análise do CTC de um produto, ou seja, o CCV é exposto como justificativa ou como meio para o CTC.

Frente à problematização apresentada, surge a seguinte questão de pesquisa: Quais as diferenças e semelhanças entre o CTC e o CCV? Convergindo a este propósito, o objetivo geral é analisar as diferenças e as semelhanças entre o CTC e o CCV.

Este trabalho é importante, pois visa contribuir com informações resultantes de reflexões e de análises das definições desses dois artefatos da GEC, a partir da literatura existente. Os resultados podem representar suporte a novas pesquisas, possibilitando uma redução dos possíveis transtornos causados por conta da confusão em sua diferenciação e compreensão.

Além desta introdução, o artigo se divide em mais quatro seções. A segunda seção apresenta o referencial teórico, que trata das definições de CTC e do CCV objetivando a compreensão da problemática. Após, são apresentados os procedimentos metodológicos que compõem o estudo. Na quarta seção é apresentada a discussão dos resultados da pesquisa juntamente com as devidas análises. Por fim, a última seção traz as conclusões e sugestões para trabalhos futuros. 


\section{Referencial teórico}

\subsection{Custeio do ciclo de vida do produto (CCV)}

O planejamento do ciclo de vida dos produtos vem ganhando cada vez mais espaço dentro e fora das organizações, o que induz a mudanças nos sistemas praticados. A partir dos anos de 1980 e 1990, as pesquisas começaram se voltar para formas de redução de custos, o que levou a análises do custo de todo o ciclo de vida de um produto, até o descarte (YAO, JIA; MAO, 2010). A redução dos ciclos de vida dos produtos, aliado à necessidade econômica de aumento de resultados e consequente avaliação de custos, impulsionou uma maior atenção dessa ferramenta perante o tomador de decisões ao longo da vida de um bem (MIAH, KOH; STONE, 2017).

O CCV é, assim, uma das respostas às novas demandas impostas pelo mercado, compondo um artefato da GEC e conquistando espaço a partir da influência do ambiente global. Esse embate impulsionou as empresas a uma constante criação e renovação dos produtos, visto a velocidade com que são substituídos pelos consumidores e a redução de seu ciclo de vida, quer seja por conta da tecnologia, desenho, formato, diferenciais, dentre outros vários fatores (WERNKE, 2004).

Cabe ressaltar que a visão do ciclo de vida do produto não representa algo inovador, pois já era explorado pelas empresas. Entretanto, a abordagem era limitada ao controle dos custos de produção e de aquisição, o que é praticado na maioria das empresas ainda hoje, como cita Sakurai (1997). As informações de custos tradicionais já ofereciam informações sobre o ciclo de vida do produto, porém eram levados em conta os custos, que compreendiam desde a fabricação até a entrega ao consumidor. A GEC amplia essa abordagem considerando como custo também as fases da concepção até o descarte, em que o produto perde sua utilidade ou se desgasta totalmente, incluindo também custos com armazenagem, transporte, energia, mão de obra, treinamento, manutenção, dentre outros (WERNKE, 2004).

A Figura 1 mostra as etapas relevantes do CCV do Produto, iniciando no projeto e terminando com o descarte. Essa visão engloba a abordagem atual relativa a este artefato de maneira ampla.

Essa visão do CCV é essencial frente às avaliações dos ciclos de vida dos produtos, uma vez que as chances de análises e de descobertas de oportunidades aumentam, quando se passa a olhar desde as fases de planejamento, e o suporte informacional inclui mais áreas, possibilitando, por exemplo, informações sobre o processo de utilização, custo-benefício da aquisição, ou decisões voltadas à economia de dinheiro em longo prazo (YAO, JIA; MAO, 2010). A moderna concepção reporta a análise dos custos do ciclo de vida do produto muito antes da fase inicial produtiva, em fases precedentes, uma vez que essas etapas concentram a grande maioria dos custos de toda a vida útil de um produto (ZACHARIASSEN; ARLBJORN, 2011). 
Figura 1: Ciclo de vida completo do produto

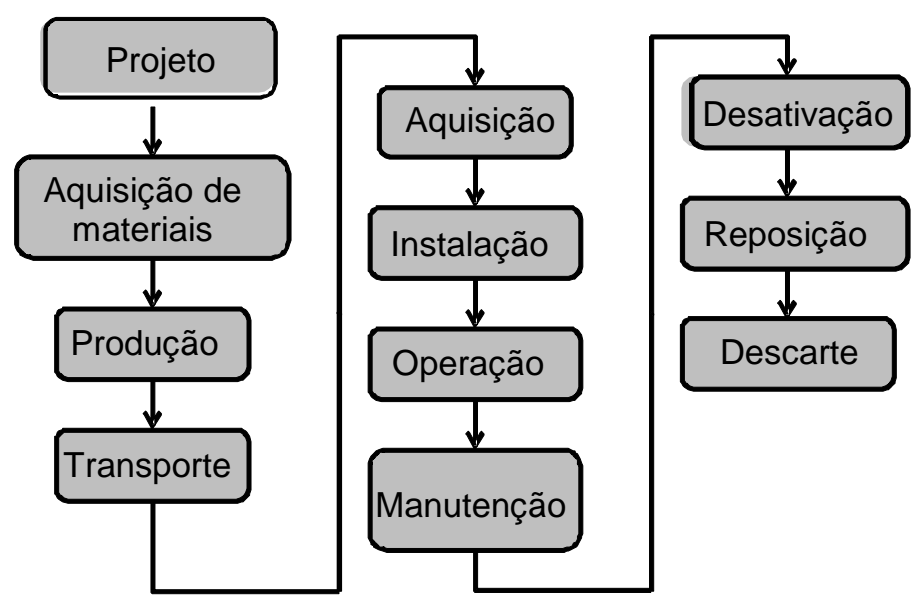

Fonte: Santos e Tenório (2010).

Para ser competitivo no mercado atual, "o fabricante deve desenhar um produto que, desde o início, tenha qualidade, confiabilidade e assistência, para poder otimizar o desempenho e a lucratividade do usuário" (SAKURAI, 1997, p. 159). A forma mais vantajosa de reduzir o custo total do ciclo de vida é observar os custos que ocorrem antes do início da produção, ou seja, os gastos com P\&D (SAKURAI, 1997). Destarte, analisando as etapas do ciclo de vida e focando na abrangência oferecida pela GEC, é possível perceber a necessidade de uma maior atenção nas fases precedentes à produção, como no desenho e projeto, já que nesse período é que são comprometidos a maior parte dos custos totais do produto (SHIELDS; YOUNG, 1991).

Em relação às vantagens desse artefato, Okano (2001) destaca o oferecimento de importantes informações sobre design e operação do produto, otimização dos projetos e avaliação de alternativas de melhorias, eliminação de atividades que não agregam valor, e maior satisfação do consumidor, quando em contato com o produto ou serviço. Já Berliner e Brimson (1992) citam como vantagem o oferecimento de um quadro claro de rentabilidade do produto em longo prazo; eficiência no planejamento de todo o ciclo de vida e possibilidade de quantificar o impacto de custos da alternativa escolhida na fase de P\&D.

A relevância de um olhar mais direto aos possíveis custos futuros pode agregar valores tanto à empresa quanto ao consumidor. No que diz respeito ao usuário, Wernke (2004) enfatiza a contribuição para análises de alternativas visando decisão de compra por um determinado produto, considerando todos os gastos inclusos no ciclo de vida da proveniente aquisição. O mesmo autor enxerga neste artefato uma busca pela eficácia da empresa, por meio da compreensão de quanto custa para o cliente ter o produto.

A seguir é apresentado o CTC. Tanto o CCV como o CTC se entrelaçam, em alguns momentos, o que dificulta a realização de análises do CTC sem antes conhecer os estágios do ciclo de vida de um determinado produto e não há como gerir, estrategicamente, o ciclo de vida sem conhecer os custos, que este produto ou serviço acarretará para o consumidor (SOUTES, 2007). 


\subsection{Custo total para o consumidor (CTC)}

O cenário competitivo obriga as empresas à constante busca por oportunidades de melhor análise de custos perante a cadeia de valor, preferivelmente, em longo prazo, e com otimização de capacidade preditiva, que sejam capazes de subsidiar as decisões de compra, atmosfera essa na qual se intensifica o uso de práticas relacionadas ao CTC (MENDES, BARBOSA NETO; DIAS, 2012).

Decisões de compra levando em conta somente o preço de aquisição representam análises incompletas e podem gerar prejuízos e, assim, tais avaliações devem incluir os custos envolvidos no horizonte temporal ocupado pelo produto, mesmo que por meio de informações estimadas (SACCANI, PERONA; BACCHETTI, 2017), atitude que otimiza resultados e mostra que nem sempre o mais barato é a melhor alternativa.

Assim, "este artefato consiste em reconhecer e mensurar os custos que um produto apresenta a partir de sua compra, incluindo os custos de aquisição, utilização, manutenção e descarte e custos com produtos complementares, necessários ao funcionamento do produto principal" (SILVA ET AL., 2015, p. 145). A utilidade principal se refere à forma de antever os custos a incorrer durante a vida útil do bem, bem como sua relevância pela possibilidade de prática pelas mais diversas áreas e para os mais variados tipos de produtos mesmo que, inicialmente, a literatura só tratava de bens de capital (SOUTES, 2007).

Amaral e Souza (2016) citam como diferenciais, primeiramente, a questão do CTC ser uma arma poderosa para as empresas, tanto das decisões de compra, quanto de vendas. Por outro lado, também se sobressai, nesse contexto, a utilidade perante o consumidor, que pode avaliar mais profundamente suas escolhas, aprimorando as análises em uma perspectiva de longo prazo. E por fim, ressaltam o diferencial para os fornecedores, representando uma ferramenta gerencial para análises do produto que oferecem a seus clientes (AMARAL; SOUZA, 2016).

Silva et al. (2015) acrescentam que com uma maior ênfase nas análises que envolvem o CTC, a empresa tende a agir de modo mais estratégico, com um olhar em longo prazo, e com as decisões de compras, bem como suas consequências, como parte de seu planejamento estratégico. Por outro lado, esse artefato, sob a ótica do consumidor, pode contribuir para suas decisões de compra, uma vez que frente à variedade de ofertas torna-se vantajoso um suporte seguido de análises críticas de informações e de avaliações de custos (SILVA ET AL., 2015).

Destarte, o CTC atua como uma ferramenta de análise essencial ao novo contexto competitivo, tanto em relação à empresa que produz o produto, quanto ao consumidor que adquire este produto, pois demonstra na totalidade, os impactos financeiros causados pela aquisição do produto e, também, os custos a incorrer em toda sua vida útil, possibilitando uma visão ampla em longo prazo e o suporte para decisões de compra mais sensatas.

Uma maneira simples de entendimento quanto ao funcionamento desse artefato estratégico é trazido por Sakurai (1997): primeiramente, os custos relevantes devem ser identificados; depois, o fluxo futuro de caixa deve ser analisado e, finalmente, esse fluxo de caixa deve ser trazido a valor presente. Dessa forma, o tomador de decisão estará amparado para analisar e escolher o melhor produto com base em uma ferramenta de visão holística. 
Já que o CTC é uma ferramenta de análise estratégica de custos, é aceitável que esses custos sejam divididos e categorizados. Ellram e Siferd (1998) tratam que essas categorias, basicamente, devem abranger as etapas de aquisição, utilização e manutenção do produto. Posteriormente, Amaral e Souza (2016) apresentam um quadro expondo essas categorias, mais as etapas de transporte, de instalação, de propriedade e de descarte, conforme demonstrado no Quadro 1.

\section{Quadro 1: Tipos de custos do consumidor}

\begin{tabular}{|c|c|c|c|}
\hline \multirow{2}{*}{$\begin{array}{l}\text { CATEGORIA } \\
\text { AQUISIÇÃO }\end{array}$} & \multicolumn{3}{|c|}{ EXEMPLOS DE CUSTOS EM CADA CATEGORIA } \\
\hline & $\begin{array}{l}\text { Preço de compra da } \\
\text { unidade do produto }\end{array}$ & $\begin{array}{c}\text { Preço de possíveis } \\
\text { produtos } \\
\text { complementares }\end{array}$ & $\begin{array}{c}\text { Custos de } \\
\text { financiamento voltados } \\
\text { ao pagamento }\end{array}$ \\
\hline TRANSPORTE & Possível tarifa de frete & $\begin{array}{l}\text { Impostos sobre a } \\
\text { tarifa de frete }\end{array}$ & $\begin{array}{l}\text { Tempo e insumos (por } \\
\text { exemplo: combustível, } \\
\text { em caso de transporte } \\
\text { com veículo próprio) }\end{array}$ \\
\hline INSTALAÇÃO & $\begin{array}{l}\text { Tarifa de instalação da } \\
\text { unidade de produto }\end{array}$ & $\begin{array}{l}\text { Impostos sobre a } \\
\text { tarifa de instalação }\end{array}$ & $\begin{array}{c}\text { Tempo e insumos (por } \\
\text { exemplo: combustível } \\
\text { gasto) }\end{array}$ \\
\hline PROPRIEDADE & $\begin{array}{l}\text { Impostos decorrentes } \\
\text { da propriedade }\end{array}$ & $\begin{array}{c}\text { Seguro e garantia } \\
\text { estendida do } \\
\text { produto }\end{array}$ & $\begin{array}{l}\text { Tarifas decorrentes da } \\
\text { propriedade (como, por } \\
\text { exemplo, condomínios) }\end{array}$ \\
\hline UTILIZAÇÃO & $\begin{array}{l}\text { Tempo e insumos } \\
\text { (como por exemplo } \\
\text { energia elétrica) com a } \\
\text { utilização do produto }\end{array}$ & $\begin{array}{l}\text { Consumo de } \\
\text { combustível e } \\
\text { insumos }\end{array}$ & $\begin{array}{c}\text { Preço dos produtos } \\
\text { complementares } \\
\text { utilizados. }\end{array}$ \\
\hline MANUTENÇÃO & $\begin{array}{c}\text { Manutenção } \\
\text { preventiva da unidade } \\
\text { do produto }\end{array}$ & $\begin{array}{c}\text { Manutenção } \\
\text { corretiva da } \\
\text { unidade do produto }\end{array}$ & - \\
\hline DESCARTE & $\begin{array}{l}\text { Possível tarifa de } \\
\text { descarte do produto }\end{array}$ & $\begin{array}{l}\text { Impostos sobre a } \\
\text { tarifa de descarte }\end{array}$ & $\begin{array}{c}\text { Tempo e insumos } \\
\text { (como, por exemplo, } \\
\text { combustível) }\end{array}$ \\
\hline
\end{tabular}

Fonte: Amaral e Souza (2016)

Como fatores condicionantes para a aplicação do CTC devem ser levados em consideração, segundo Silva et al. (2015): conhecimento sobre o produto principal; conhecimento sobre os produtos complementares; especificidades do uso (frequência, tempo, ambiente) e a interação do CTC com o planejamento estratégico do cliente. Entretanto, como fatores que impedem a eficácia do CTC tem-se o fato de muitas organizações terem dificuldades para caracterizar os custos inclusos para os cálculos, bem como quanto ao modo como devem realizar os custos estimados até o fim da vida útil do produto (SACCANI, PERONA; BACCHETTI, 2017). Também cabe enfatizar a pouca atenção dada à formalização e ao tratamento das atividades e recursos necessários para a operacionalização, e as dificuldades em implantar e entender os modelos propostos para a prática do artefato nas organizações (SACCANI, PERONA; BACCHETTI, 2017).

Esse artefato, portanto, compõe parte das opções disponíveis à busca do gerenciamento estratégico de custos, frente ao mercado competitivo e à necessidade por vantagem competitiva. Por ser um assunto recente, a literatura ainda propõe 
modelos e formas de executá-lo, talvez nem sempre claros e eficazes, mas sempre enfatizando o objetivo final à empresa e ao consumidor, principalmente.

\section{Metodologia}

Diante da problemática e objetivos propostos, esse artigo se caracteriza como uma análise crítico-interpretativa da literatura, destacando-se a discussão das definições presentes nas pesquisas com o objetivo de diferenciar os dois artefatos em questão.

Como este estudo não apresenta nenhuma relação com modelos estatísticos, a abordagem do problema se classifica como qualitativa, sendo aquela associada à coleta de informações, observações e análises de estudos sobre determinado assunto (SILVA, 2010).

Os dados para o estudo foram coletados a partir de trabalhos encontrados na literatura nacional e internacional existente sobre o CCV e CTC.

\section{Apresentação e análise dos resultados}

\subsection{CTC: evolução da definição}

Definições se modelam através dos pensamentos e estudos dos autores. Interessante também é que cada autor traz um fragmento de individualidade e percepção pessoal. Amaral e Souza (2016) enfatizam que existe uma falta de consenso no refinamento das definições de CTC e, assim, não se pode enxergar uma hegemonia do conceito ao descrever tal artefato. Foram analisados trabalhos nacionais e internacionais sobre o CTC, a fim de expor a construção da sua definição ao longo do tempo, o que será a base para as reflexões a serem desenvolvidas em tópico posterior, conforme apresenta o Quadro 2.

\section{Quadro 2: Levantamento das definições de CTC}

\begin{tabular}{|c|l|l|}
\hline Autor (ano) & \multicolumn{1}{|c|}{ Definição original } & $\begin{array}{l}\text { Definição em português (tradução } \\
\text { livre) }\end{array}$ \\
\hline CARR E ITTNER (1992) & $\begin{array}{l}\text { Structured approach to determine } \\
\text { the total costs associated with the } \\
\text { acquisition and further use of a } \\
\text { given good or service from a given } \\
\text { supplier. }\end{array}$ & $\begin{array}{l}\text { Abordagem estruturada para } \\
\text { determinar os custos totais } \\
\text { associados à aquisição e ao posterior } \\
\text { uso de um dado bem ou serviço de } \\
\text { um dado fornecedor. }\end{array}$ \\
\hline $\begin{array}{c}\text { DEGRAEVE, LABRO E } \\
\text { ROODHOOFT (2000, p. } \\
\text { 35) }\end{array}$ & $\begin{array}{l}\text { TCO quantifies all costs } \\
\text { associated with the purchasing } \\
\text { process throughout the entire } \\
\text { value chain of the firm. }\end{array}$ & $\begin{array}{l}\text { CTC quantifica todos os custos } \\
\text { associados com o processo de } \\
\text { aquisição do produto, por meio de } \\
\text { toda a cadeia de valor da firma. }\end{array}$ \\
\hline
\end{tabular}




\begin{tabular}{|c|c|c|}
\hline $\begin{array}{c}\text { ELLRAM; } \\
\text { ZSIDISIN (2002, p. 270) }\end{array}$ & $\begin{array}{l}\text { TCO analysis is a structured } \\
\text { approach for determining the total } \\
\text { costs associated with acquiring a } \\
\text { good or service. Many of the costs } \\
\text { analyzed and eventually reduced } \\
\text { or eliminated by purchasing } \\
\text { organizations using a TCO model } \\
\text { can be classified as transaction } \\
\text { costs }\end{array}$ & $\begin{array}{l}\text { A análise de CTC é uma abordagem } \\
\text { estruturada para a a das } \\
\text { determinação dos custos totais } \\
\text { associados com a aquisição de um } \\
\text { bem ou serviço. Muitos dos custos } \\
\text { analisados e, eventualmente, } \\
\text { reduzidos ou eliminados por } \\
\text { organizações de compra utilizando } \\
\text { um modelo CTC podem ser } \\
\text { lassificados como custos de } \\
\text { transação. }\end{array}$ \\
\hline $\begin{array}{l}\text { WOUTERS } \\
\text { et. al. }(2005, \text { p. } 1)\end{array}$ & $\begin{array}{l}\text { TCO is a cost accounting } \\
\text { application that enables } \\
\text { purchasing decision-makers to } \\
\text { combine value and price in } \\
\text { making sourcing decisions. }\end{array}$ & $\begin{array}{l}\text { TCO é uma aplicação da } \\
\text { contabilidade de custos, que permite } \\
\text { tomar decisões } \\
\text { compra combinado valor e preço. }\end{array}$ \\
\hline $\begin{array}{c}\text { HEILALA, } \\
\text { MONTONEN E HELIN } \\
(2007, \text { p. } 47)\end{array}$ & $\begin{array}{l}\text { TCO includes all costs, direct and } \\
\text { indirect, incurred throughout the } \\
\text { life cycle of an asset, including } \\
\text { acquisition and procurement, } \\
\text { operations and maintenance, and } \\
\text { end-life management. }\end{array}$ & $\begin{array}{l}\text { CTC inclui todos os custos, diretos e } \\
\text { indiretos, incorridos em todo o ciclo } \\
\text { de vida de um ativo, incluindo } \\
\text { aquisição e contratação, operações e } \\
\text { manutenção, e gestão do fim de vida. }\end{array}$ \\
\hline $\begin{array}{l}\text { ZACHARIASSEN E } \\
\text { ARLBJORN (2011) }\end{array}$ & $\begin{array}{l}\text { The common denominator for } \\
\text { TCO definitions seems to be the } \\
\text { use of TCO as a way of focusing } \\
\text { on the indirect procurement costs } \\
\text { and life-cycle costs related to } \\
\text { carrying out transactions with } \\
\text { suppliers. }\end{array}$ & $\begin{array}{l}\text { O denominador comum para } \\
\text { definições de TCO aparenta ser o uso } \\
\text { deste como uma maneira de focar } \\
\text { nos custos indiretos de aquisição e } \\
\text { ciclo de vida relacionados às } \\
\text { transações com fornecedores. }\end{array}$ \\
\hline $\begin{array}{c}\text { MORSSINKHOF, } \\
\text { WOUTERS E } \\
\text { WARLOP }(2011, \text { p. 132) }\end{array}$ & $\begin{array}{l}\text { Total cost of ownership (TCO) } \\
\text { involves the monetary } \\
\text { quantification of nonfinancial } \\
\text { attributes and the subsequent } \\
\text { aggregation of these attributes } \\
\text { into a financial summary measure }\end{array}$ & $\begin{array}{l}\text { CTC envolve a quantificação } \\
\text { monetária de atributos não } \\
\text { financeiros e a subsequente } \\
\text { agregação desses atributos em um } \\
\text { resumo de medida financeira. }\end{array}$ \\
\hline $\begin{array}{l}\text { MENDES, BARBOSA } \\
\text { NETO E DIAS (2012, p. } \\
\text { 92) }\end{array}$ & $\begin{array}{l}\text { An integration concept, being } \\
\text { determined from the activity flow } \\
\text { relative to the purchase of a good } \\
\text { or service and the costs } \\
\text { associated with these activities. }\end{array}$ & $\begin{array}{l}\text { Um conceito de integração, sendo } \\
\text { determinado a partir do fluxo de } \\
\text { atividades relativas à compra do bem } \\
\text { ou serviço e dos custos associados a } \\
\text { essas atividades. }\end{array}$ \\
\hline $\begin{array}{l}\text { SCHMIDT ET AL }(2013, \\
\text { p. } 21)\end{array}$ & $\begin{array}{l}\text { TCO is an analysis tool for the } \\
\text { acquisition of goods and services } \\
\text { of a specific supplier. }\end{array}$ & $\begin{array}{l}\text { TCO é uma ferramenta de análise de } \\
\text { custos de aquisição de bens e } \\
\text { serviços de um determinado } \\
\text { fornecedor. }\end{array}$ \\
\hline $\begin{array}{c}\text { SILVA ET AL. }(2015, \mathrm{P} . \\
153)\end{array}$ & & $\begin{array}{l}\text { Artefato que abrange todos os custos } \\
\text { que o consumidor ou cliente terá, } \\
\text { decorrente da compra de um } \\
\text { determinado produto junto a um } \\
\text { fornecedor, desde a sua aquisição } \\
\text { até o seu descarte, incluindo custos } \\
\text { com produtos complementares } \\
\text { necessários ao funcionamento do } \\
\text { produto principal. }\end{array}$ \\
\hline
\end{tabular}

Fonte: Elaborado pelos autores com base nos autores citados. 
Cabe citar que não foi elencada a visão de todos os autores que exploram o tema, mas se procurou buscar definições de forma a possibilitar uma visualização cronológica e construtiva do que é o artefato. Foram encontrados trabalhos internacionais desde meados do ano 1992, como o estudo de Carr e Itner, se referindo à aplicação dos princípios do CTC na cadeia de suprimentos. Em nível nacional se destaca como um dos pioneiros no assunto o autor Welington Rocha, por meio de sua tese de doutorado no ano de 1999, na qual abordava aspectos relacionados a sistemas e informações na gestão estratégica.

A essência das definições analisadas, de forma geral, aborda o CTC como predominante no processo de aquisição de um produto, bem como de seus custos posteriores. Somente Heilala, Montonen e Helin (2007) acrescentam aspectos que reportam a todos os custos incorridos no ciclo de vida de um ativo, porém não especificam onde iniciam; Morssinkhof, Wouters e Warlop (2011), que incluem atributos não financeiros, cabendo questionar o que seriam esses atributos e de que forma seriam inclusos nos cálculos e análises do CTC; e Mendes, Barbosa Neto e Dias (2012), que enfatizam os custos relativos às atividades realizadas, além de todos os custos relativos a essas atividades, porém cabe questionar: até em que fase? Tal ponto não fica claro. A seguir, é apresentado um quadro similar do CCV. Posteriormente, iniciam-se as reflexões conjuntas entre as definições.

\subsection{CCV: evolução da definição}

Da mesma forma, buscou-se definições do CCV, de modo a conduzir uma construção da definição ao longo do tempo e representar uma base para as reflexões posteriores sobre o tema, conforme apresentado no Quadro 3.

\section{Quadro 3: Levantamento das definições do custeio por ciclo de vida}

\begin{tabular}{|c|c|c|}
\hline Autor (ano) & Definição original & $\begin{array}{l}\text { Definição em português } \\
\text { (tradução livre) }\end{array}$ \\
\hline ELLRAM (1995, p. 5) & $\begin{array}{l}\text { Life-cycle costing focuses } \\
\text { primarily on capital or fixed } \\
\text { assets. The emphasis is } \\
\text { understanding the purchase price } \\
\text { of the asset and also determining } \\
\text { how much it actually costs the } \\
\text { organization to use, maintain and } \\
\text { dispose of that asset during its } \\
\text { lifetime. Pre-transaction costs } \\
\text { tend to be deemphasized. The } \\
\text { life-cycle approach is congruent } \\
\text { with TCO, but represents only a } \\
\text { subset of TCO activity. TCO is } \\
\text { broader in scope and includes the } \\
\text { pre-purchase costs associated } \\
\text { with a particular supplier }\end{array}$ & $\begin{array}{l}\text { O custeio por ciclo de vida foca, } \\
\text { primariamente, em ativos imobilizados } \\
\text { ou de capital. A ênfase é compreender } \\
\text { o preço de compra de um ativo e } \\
\text { também determinar quanto de fato } \\
\text { custará para organizar, usar, manter, e } \\
\text { descartar esse determinado ativo } \\
\text { durante sua vida. Custos pré- } \\
\text { transacionais tendem a não ser } \\
\text { enfatizados. A abordagem do ciclo de } \\
\text { vida é congruente com o CTC, mas } \\
\text { representa apenas uma parte da } \\
\text { atividade de CTC. O CTC é mais } \\
\text { amplo em escopo e inclui os custos } \\
\text { pré-aquisição associados com a } \\
\text { escolha de um determinado } \\
\text { fornecedor. amado a }\end{array}$ \\
\hline SAKURAI (1997, p. 157) & & $\begin{array}{l}\text { Método de apuração do custo de um } \\
\text { produto até o fim de sua vida útil. }\end{array}$ \\
\hline
\end{tabular}




\begin{tabular}{|c|c|c|}
\hline $\begin{array}{c}\text { ELLRAM E } \\
\text { SIFERD }(1998, \text { p. 57) }\end{array}$ & $\begin{array}{l}\text { Life-cycle costing focuses } \\
\text { primarily on capital or fixed } \\
\text { assets. The aim is to go beyond } \\
\text { the purchase price of an asset, to } \\
\text { determine how much it actually } \\
\text { costs the organization to use, } \\
\text { maintain, and dispose of that } \\
\text { asset during its lifetime. }\end{array}$ & $\begin{array}{l}\text { O custeio por ciclo de vida foca, } \\
\text { prioritariamente, em ativos } \\
\text { imobilizados ou de capital. O objetivo } \\
\text { é ir além do preço de compra de um } \\
\text { ativo, para determinar quanto } \\
\text { realmente custa para a organização } \\
\text { usar, manter, e descartar esse ativo } \\
\text { durante o tempo de vida. }\end{array}$ \\
\hline $\begin{array}{c}\text { OKANO }(2001, \text { p. } 317- \\
318)\end{array}$ & $\begin{array}{l}\text { Life-cycle Costing (LCC) analysis } \\
\text { is a method of economic } \\
\text { evaluation of alternatives which } \\
\text { considers all relevant costs (and } \\
\text { benefits) associated with each } \\
\text { alternative activity or project over } \\
\text { its life. [...] Ideally should start at } \\
\text { the product's inception and should } \\
\text { be expanded to take into account } \\
\text { all the costs that will be incurred } \\
\text { throughout its lifetime. }\end{array}$ & $\begin{array}{l}\text { A análise do custo por ciclo de vida } \\
\text { (CCV) é um método de avaliação } \\
\text { econômica das atividades que } \\
\text { considera todos os custos (e } \\
\text { benefícios) associados com cada } \\
\text { atividade alternativa ou projeto durante } \\
\text { sua vida. [...] idealmente deve } \\
\text { começar no início do produto e deve } \\
\text { ser expandido para englobar todos os } \\
\text { custos que serão incorridos ao longo } \\
\text { do tempo de vida. }\end{array}$ \\
\hline $\begin{array}{c}\text { DIAS FILHO, } \\
\text { NAKAGAWA E ROCHA } \\
\text { (2002) }\end{array}$ & & $\begin{array}{l}\text { Instrumento de apoio à } \\
\text { competitividade dos negócios que visa } \\
\text { identificar todos os custos, até o fim da } \\
\text { vida útil do produto. }\end{array}$ \\
\hline $\begin{array}{l}\text { HANSEN E MOWEN } \\
(2010 \text {, p. 439) }\end{array}$ & & $\begin{array}{l}\text { Consiste em ações tomadas que } \\
\text { provocam projeção, desenvolvimento, } \\
\text { produção, comercialização, } \\
\text { distribuição, operações, manutenção, } \\
\text { atendimento e descarte de um produto } \\
\text { para que os lucros do ciclo de vida } \\
\text { sejam maximizados. }\end{array}$ \\
\hline YAO, JIA E MAO (2010) & $\begin{array}{l}\text { Life cycle is the whole process of } \\
\text { a product throughout the raw } \\
\text { material acquisition, production, } \\
\text { application and eventually } \\
\text { abandonment, and life cycle cost } \\
\text { (LCC) is the calculation of all the } \\
\text { products' expenses throughout } \\
\text { the life cycle. }\end{array}$ & $\begin{array}{l}\text { O ciclo de vida é todo o processo de } \\
\text { um produto ao longo da aquisição da } \\
\text { matéria-prima, produção, aplicação e, } \\
\text { eventualmente, o abandono, e o } \\
\text { custeio por ciclo de vida é o cálculo de } \\
\text { todas as despesas de um produto ao } \\
\text { longo do ciclo de vida. }\end{array}$ \\
\hline $\begin{array}{c}\text { ILG, HOEHNE E } \\
\text { GUENTHER (2016, p. } \\
\text { 928) }\end{array}$ & $\begin{array}{l}\text { An economic method for project } \\
\text { assessment and evaluation of all } \\
\text { (direct, indirect, internal, and } \\
\text { external) costs and revenues } \\
\text { arising within a defined life cycle. }\end{array}$ & $\begin{array}{l}\text { Um método econômico para avaliação } \\
\text { de projetos e avaliação de todos } \\
\text { custos (direto, indireto, interno, } \\
\text { externo) e receitas advindas dentre de } \\
\text { um ciclo de vida definido. }\end{array}$ \\
\hline
\end{tabular}

Fonte: Elaborado pelos autores

Ressalta-se que não foram apresentadas no Quadro 3 todas as definições sobre o assunto, mas algumas visando possibilitar sua compreensão e evolução. Foi encontrado um número mais significativo de trabalhos em âmbito internacional, mas cabe citar que, em comparação ao CTC, foi observado um número inferior de estudos no geral. 
As definições apresentadas, independente da ordem cronológica, tendem a algo de cunho geral, compreendendo os estágios e os custos de toda a vida útil do produto, sem maiores explicações de como é feito ou de qual seu propósito. A definição de Ellram (1995) e Ellram e Siferd (1998) chama a atenção por destacar os custos anteriores à aquisição e custos posteriores, o que confunde com o CTC. Estes autores chegam a mencionar que o CTC é mais abrangente que o $\mathrm{CCV}$, aspecto que será discutido mais detalhadamente no tópico a seguir.

\subsection{Em busca de uma diferenciação clara}

Vários estudos, principalmente, em nível internacional, abordam o CTC e o CCV nos mais diversos campos como o meio empresarial, área automobilística e informática. Percebe-se a preocupação em diferenciar os dois artefatos na literatura, porém muitas vezes existe falta de clareza nesse intento. Assim, busca-se neste tópico a discussão da abordagem de alguns autores quanto às diferenças existentes entre os dois artefatos, bem como as reflexões a partir dos respectivos pontos de vista.

Em consonância com o aspecto temporal e devido ao destaque conquistado, em meio a literatura, será abordada primeiramente a definição de Ellram (1995), segundo a qual o CTC e o CCV são artefatos que coincidem em várias características, caminhando juntos, porém ressalta que o CTC é mais abrangente por conta da inclusão de mais fatores, que envolvem os custos de um produto (ELLRAM, 1995), conforme Quadro 3. Na tentativa de demonstrar o ponto de vista apresentado pela autora se apresenta a Figura 2.

\section{Figura 2: CTC e CCV Segundo Ellram}

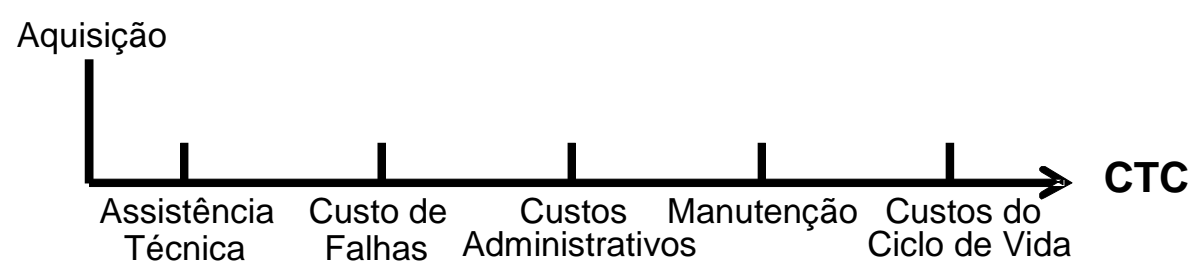

Fonte: Adaptado de Ellram (1995)

Pelo fato do CTC ser definido como mais abrangente e por ficar claro que os dois artefatos possuem características muito semelhantes, em essência, conforme Ellram (1995), coube considerar os custos do ciclo de vida como parte do CTC na tentativa de representação visual da definição. Ellram (1995) também destaca que o CCV foca, principalmente, os ativos fixos ou bens de capital, já o CTC é tratado como uma ferramenta de compra, independente do tipo do produto/serviço a ser adquirido de um fornecedor.

Em 1998, a definição apresentada é de que o CTC é uma ferramenta de compra que visa a compreensão do custo relativo à aquisição e consequente utilização de um produto adquirido de determinado fornecedor; mostra-se como uma abordagem complexa, compreendendo não só o preço de venda, mas também inclui custos de posse, de uso e de descarte subsequente de um bem ou serviço (ELLRAM; SIFERD, 1998). Já no CCV, o "objetivo é ir além do preço de compra de um ativo, para 
determinar quanto realmente custa para a organização usar, manter, e descartar esse ativo durante o tempo de vida" (ELLRAM; SIFERD, 1998, p. 57).

Percebe-se, claramente, que as duas definições tangem quase que exatamente à mesma essência e objetivo, ou seja, o de apurar os custos relativos à aquisição do produto e os possíveis custos a ocorrerem posteriormente, até o fim de sua vida útil. No entanto, afinal, no que se diferem estes dois artefatos?

\section{Figura 3: CCV e CTC segundo Ellram e Siferd (1998)}

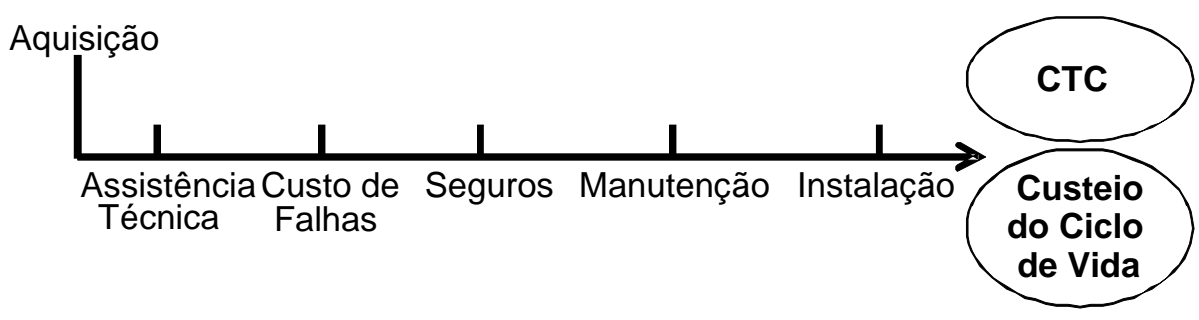

Fonte: Adaptado de Ellram e Siferd (1998)

Analisando a Figura 3, bem como as definições de Ellram e Siferd (1998) percebe-se que a abordagem do CTC e do CCV englobam o preço de aquisição, mais os gastos possíveis a surgir com o consumo do bem em sua vida útil. Pode-se efetuar uma análise adicional supondo a menção do CCV e do CTC com a mesma essência, partindo da premissa dos custos do ciclo de vida anterior à venda estarem embutidos no preço de aquisição. Porém, isso não fica claro diante das definições expostas.

Cabe ainda enfatizar que, principalmente, os trabalhos da autora de 1995 e 2002 definem estritamente a essência do CTC, enquanto artefato de compra com foco na identificação dos custos totais provenientes da escolha de um produto, o que poderia ser visualizado, claramente, pela Figura 3. Desta forma, o início do uso do artefato ocorre na aquisição do produto até o descarte final.

Já Sakurai (1997) define o CCV como sendo um método para apurar os custos de um produto durante toda sua vida útil. No entanto, o que significa vida útil? Quando começa e termina? Em busca desse significado recorreu-se ao Dicionário Michaelis. A palavra "vida" representa a existência e durabilidade das coisas ou ainda o espaço de tempo entre o nascimento e morte de algo. A palavra "útil" é definida como algo que tem uso, ou que serve para alguma coisa. Desse modo, a definição de vida útil poderia ser expressa como: o período da existência do produto, ou seja, desde sua origem (P\&D) até o descarte, ou seja, sua extinção.

Assim, a definição de Sakurai deixa dúvidas se a vida útil se refere a todos os custos desde a concepção do produto até seu descarte ou se é a vida útil de utilização do produto. No entanto, depois de definir o CCV, Sakurai (1997) menciona que esse possui duas categorias: os custos de produção e os custos do usuário. Desse modo, se reforça o entendimento de que a vida útil se estende da concepção ao descarte do bem. A definição de Sakurai (1997) também é compartilhada por Hansen e Mowen (2010).

Em relação ao CTC, Degraeve, Labro e Roodhooft (2000) também oferecem uma definição com falta de clareza: O CTC quantifica todos os custos associados ao processo de aquisição do produto, por meio de toda a cadeia de valor da firma. 
Quando é mencionado "toda a cadeia de valor da firma" pode-se entender que a abrangência atinge desde o início do processo produtivo, e isso, segundo alguns autores como Sakurai (1997), se refere ao CCV e não ao CTC.

Dias Filho, Nakagawa e Rocha (2002) introduzem um tópico que busca esclarecer a relação entre o CTC e o CCV. Os autores, primeiramente, procuram mostrar a existência de um ciclo de vida gerador de receita e outro relacionado ao consumo, denominadas de vida produtora de receita e vida consumível, respectivamente. Ressalta-se o maior interesse pelo ciclo de vida gerador de receita pelo produtor, enquanto o ciclo consumível seria de interesse do cliente, sendo destacado, porém, a dependência e influência entre esses dois ciclos. Essa ideia parte da premissa afirmada por Sakurai (1997), quando separa o ciclo de vida entre produtor e consumidor. Ainda, em busca da diferenciação, após definirem estes dois ciclos de vida, os autores mencionam que "todas as atividades em conjunto (as do ciclo de vida da produção e do consumo) formam o chamado custo total para o consumidor" (DIAS FILHO, NAKAGAWA; ROCHA, 2002, p. 8). A Figura 4 é uma tentativa de representação visual das definições dos autores:

\section{Figura 4: Representação do CCV e CTC conjuntamente}

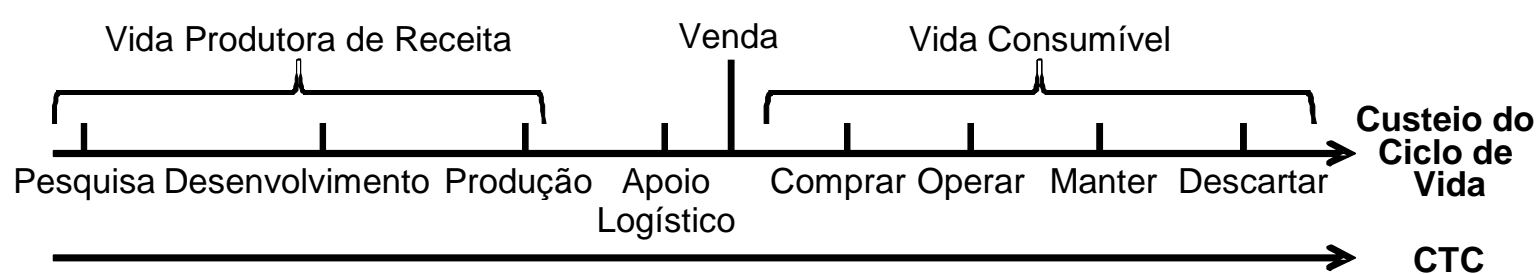

Fonte: Adaptado de Dias Filho, Nakagawa e Rocha (2002)

A partir da análise da Figura 4 percebe-se que as definições dos dois artefatos se tornam idênticas, visto que no CCV devem ser considerados os custos relativos à "Vida Produtora de Receita" e "Vida Consumível" do produto, e que a apuração do CTC corresponde à soma dos dois ciclos, conforme os referidos autores.

Outros estudos sobre CTC, como o de Wouters, Anderson e Wynstra (2005), Mendes, Barbosa Neto e Dias (2012) e Silva et al. (2015) corroboram a definição de Ellram e Siferd (1998), quando se referem ao CTC como um artefato que busca identificar e entender todos os custos relacionados com a aquisição e o consumo do produto, ou seja, abrange desde a compra até o descarte. Isso está de acordo com a Figura 3 no que se refere a representação da essência do CTC, ficando claro como ponto de partida, o ato da compra e os custos advindos do uso.

Soutes (2007, p. 91) menciona que "o custo total de propriedade parte da necessidade de se conhecer os custos que incorrerão no produto em toda sua vida útil, inclusive no momento do descarte". Essa afirmação é defendida por Sakurai (1997) na definição do CCV, conforme exposto anteriormente, tornando desta forma opostos os pontos de vista entre os dois autores em questão.

Yao, Jia e Mao (2010) também apresentam a definição do CCV, conforme Quadro 3, abrangendo desde os estágios de aquisição de matéria-prima até o possível abandono ou descarte. Nesse caso, o início da utilização do artefato está em 
desacordo com grande parte da literatura, visto que ignora aspectos como design e projeto o produto, porém o final alcança até o descarte, o que envolve a visão de grande parte das definições de CCV. Isso torna a definição incompleta frente a outros estudos na temática, conforme a Figura 5.

Figura 5: Custeio por ciclo de vida segundo Yao, Jia e Mao

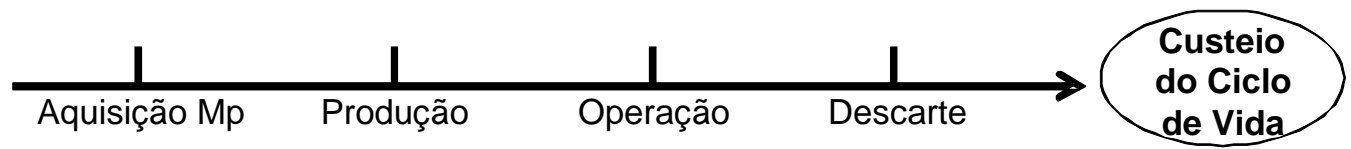

Fonte: Adaptado de Yao, Jia e Mao (2010)

Partindo das definições e das discussões expostas até aqui, em que o CCV e o CTC se cruzam, enquanto ferramentas de análise estratégica de custos? Diante da visão dos autores citados, pode-se perceber que para que os benefícios oferecidos pelo CTC sejam otimizados é necessário o conhecimento e a compreensão do ciclo de vida do produto, pois essa visão possibilitará a determinação dos custos futuros, que serão incorridos (SOUTES, 2007). A abordagem que a literatura em geral faz, intercalando os termos, pode ser entendida pela influência que os custos do início do ciclo de vida até a venda causam ao preço de venda total, que é a base para análise do CTC, impactando em toda a vida útil do bem. A exemplo desse interesse de quem produz em relação a conhecer informações do CTC, Silva et al. (2015) mencionam que conhecer informações dessa natureza é nada mais que uma busca por explorar informações estratégicas, tal como o foco do cliente e tendências, já que é este quem valida ou não o produto em questão, o que revela, consequentemente, uma fonte de vantagem competitiva.

Pelas definições dos autores citados, bem como das reflexões sobre os artefatos é apresentada a Figura 6. Buscou-se compilar os pensamentos e mostrar a diferenciação entre o CCV e o CTC, segundo o que traz a maioria dos estudos analisados.

Figura 6: Análise conjunta do custeio por ciclo de vida e CTC

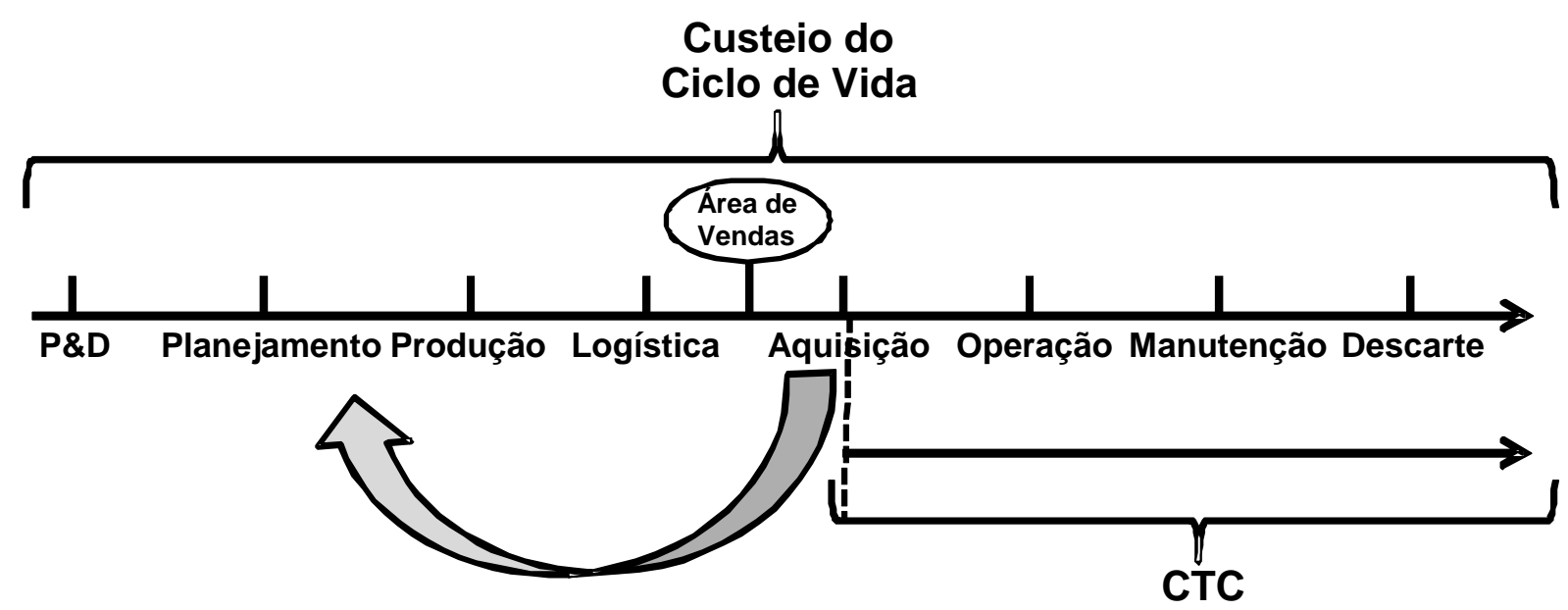

Fonte: Elaborado pelos autores

RELUNA, Belo Horizonte - MG, Brasil, v.23, n.I, p.75-96, Jan. - Mar. 2018 - ISSN 2179-8834 
Percebe-se que o custeio por ciclo de vida compreende todos os possíveis custos do produto, desde sua concepção até seu descarte final. O ponto estratégico essencial de todo o processo é o momento da aquisição, pois representa a escolha do comprador pelo produto em questão e não o do concorrente, o que reflete sua validação em relação à percepção dos esforços do produtor na redução dos custos, $\mathrm{e}$ subentende crença de maiores vantagens relacionadas aos custos possíveis totais. Anterior a essa etapa, apresenta-se o momento da produção representado na Figura 6 pelas etapas de P\&D, planejamento, produção e logística, ou seja, são os custos do fabricante.

Posteriormente ao momento da aquisição se têm as etapas normalmente devidas ao comprador, ou seja, os custos do usuário. Estas são representadas na figura pela operação, manutenção e descarte. É a partir desse ponto que se pode inferir que reside a principal diferença entre CTC e custeio por ciclo de vida. Seria nessa segunda etapa o foco do CTC. Como ponto de partida deste artefato estratégico tem-se a aquisição do produto pelo consumidor final. Todos os possíveis custos que ocorrerem após esse momento se tornam alvo de mensuração.

Fica claro, dessa forma, a abrangência do CCV e do CTC. Quanto à interrelação existente entre os dois artefatos, a representação é dada na Figura 6, por meio de uma flecha, ou seja, como a análise do CTC parte da aquisição do produto e dos possíveis custos posteriores, e partindo do pressuposto que as etapas anteriores à venda, principalmente, o desenvolvimento e planejamento, influenciam em todas as demais etapas, cabe uma análise da parte do ciclo de vida anterior ao momento da venda, pois é a partir disso que grande parte dos custos futuros ocorrerá. É importante mencionar também que, de acordo com Silva et al. (2015), o CTC tem três usuários distintos: o consumidor, o cliente e o fornecedor. Por isso, uma empresa também pode usar o CTC para gestão estratégica de suas compras de suprimentos, fazendo parte da etapa de produção no ciclo de vida do produto.

A análise conjunta dos artefatos representa uma ferramenta estratégica de alto potencial, visto que uma tem foco no consumidor em si e no impacto dos custos futuros, tentando mensurar, analisar e gerir o ônus financeiro total de um produto e serviço e, a outra contempla toda a cadeia de custos, mapeando e gerindo os custos em cada etapa do ciclo de vida, possibilitando uma análise ampla e oportuna frente ao cenário competitivo pelo qual se encontram as organizações atualmente.

Portanto, em síntese, a partir dos trabalhos analisados percebe-se que o CTC é um artefato da GEC, que abrange os efeitos de uma escolha de comprar um produto/serviço, quantificando monetariamente o ônus de propriedade desde a escolha e aquisição, estendendo-se durante toda a vida útil (enquanto tiver posse ou propriedade) até o descarte, se este último for responsabilidade do proprietário. Em contrapartida, o CCV se constitui na mensuração dos custos já realizados ou estimados ao longo dos diversos estágios, em que um produto ou serviço passa do "berço ao túmulo", com foco a obter vantagem estratégico-competitiva, oferecendo maior poder de análise para tomada de decisão e aprimoramento de imperfeições. 


\subsection{CTC versus CCV}

Diante das definições apresentadas pelos vários autores mencionados, bem como da tentativa de uma representação visual das diferenças dos dois artefatos estratégicos, foi construído o Quadro 4, que apresenta um resumo de suas características essenciais de acordo com a literatura e com as discussões expostas.

O Quadro 4 denota as principais diferenças e semelhanças entre as definições dos dois artefatos. Assim, enquanto o CTC inicia com a aquisição do produto/serviço por parte do consumidor, o custeio do ciclo de vida começa ainda na empresa, na concepção do produto. Esse é o motivo pelo qual o CTC tem um foco em custos voltado essencialmente ao "externo", enquanto o CCV se volta também ao "interno".

\section{Quadro 4: Diferenças e semelhanças entre CTC e custeio do ciclo de vida}

\begin{tabular}{|l|l|}
\hline \multicolumn{1}{|c|}{ CTC } & \multicolumn{1}{|c|}{ CCV } \\
\hline Início com a aquisição pelo consumidor. & Início na pesquisa e desenvolvimento do produto. \\
\hline Encerra com o descarte do produto. & Encerra com o descarte do produto. \\
\hline $\begin{array}{l}\text { Artefato menos amplo, representando uma parte } \\
\text { do CCV. }\end{array}$ & $\begin{array}{l}\text { Artefato mais amplo, compreendendo desde P\&D } \\
\text { até o descarte do produto. }\end{array}$ \\
\hline $\begin{array}{l}\text { Visa estimar todos os custos que podem ocorrer } \\
\text { ao longo da aquisição, uso/consumo e descarte de } \\
\text { um produto/serviço. }\end{array}$ & $\begin{array}{l}\text { Visa auferir, monitorar e controlar os custos } \\
\text { incorridos em cada fase em que o produto } \\
\text { percorre, da concepção ao descarte. }\end{array}$ \\
\hline $\begin{array}{l}\text { Essencialmente um artefato para auxiliar decisões } \\
\text { de compra. }\end{array}$ & $\begin{array}{l}\text { Essencialmente, um artefato para gestão } \\
\text { estratégica e controle de custos em cada etapa da } \\
\text { vida útil. }\end{array}$ \\
\hline $\begin{array}{l}\text { Maior interesse pelo consumidor, porém a a } \\
\text { empresa também visa conhecer os custos do } \\
\text { consumidor, representando uma ferramenta } \\
\text { estratégico-competitiva. Além disso, pode ser } \\
\text { usado para decisões de compra, aspecto também } \\
\text { de interesse da empresa. }\end{array}$ & $\begin{array}{l}\text { Interesse exclusivo da empresa com foco na } \\
\text { gestão estratégica de custos em cada etapa do } \\
\text { ciclo de vida do produto/serviço. }\end{array}$ \\
\hline
\end{tabular}

Fonte: Elaborado pelos autores

Uma característica peculiar é de que ambos os artefatos se encerram com o descarte final do produto. Todavia, não é possível presumir que o descarte sempre é por conta do consumidor final, pois existem alguns casos em que as empresas que produziram o item é que são responsáveis por essa etapa, sendo encarregadas pela reciclagem ou por direcionar o produto a um destino específico. Umas das diferenciações essenciais entre ambos, conforme a Figura 6, é que o CCV é mais amplo que o CTC de acordo com a maioria dos autores citados. Isso significa que o segundo está incluso no primeiro, mas não necessariamente que o tempo cronológico, em que o produto fica sob tutela da empresa é maior do que o tempo de uso pelo consumidor.

Por fim, cabe ainda enfatizar os interesses distintos entre os artefatos. O CTC, mesmo sendo considerado um artefato de GEC, o que de fato é, consiste em essência em uma ferramenta de compra, que contribui de forma a auxiliar o consumidor na 
opção pelo melhor produto, levando em consideração também os demais custos futuros possíveis de ocorrerem. O CCV reporta a todo o processo, tanto de produção como de consumo do produto, focando principalmente a mensuração e controle dos custos, que ocorreram e que irão ocorrer durante sua vida útil.

Conforme já citado anteriormente, atenta-se para a importância e vantagem advinda de uma análise conjunta entre esses dois artefatos. Segundo Silva et al. (2015), a análise do CTC permite fazer previsões a respeito de custos, que serão incorridos ao longo do ciclo de vida do produto/serviço, possibilitando desde melhorias no projeto do produto até a implementação de estratégias de marketing, em relação à ênfase aos baixos custos que tal produto proporcionará ao consumidor ou cliente. Desse modo, pelo CCV se tem uma visão geral dos custos, bem como o foco naqueles que ocorrem antes do momento da venda, enquanto pelo CTC se tem uma análise mais focada no período em que o produto passa para a propriedade do consumidor.

\section{Considerações finais}

A forma como o produto é construído, distribuído e consumido impulsionou grandes avanços capazes de tornar as práticas da gestão de custos compatíveis à nova realidade competitiva. Os artefatos estratégicos, que compõem a GEC buscam abranger, por meio de diferentes abordagens, a gestão de custos sob um novo olhar. Entretanto, alguns por serem recentes e com utilidade ainda limitada nas empresas possuem definições inacabadas, o que pode resultar em confusões de definições ou entendimentos discrepantes, sendo exemplos dessa situação o CCV e o CTC.

Assim, este estudo procurou, por meio da literatura existente, contribuir com os estudos sobre a GEC, mais precisamente no que compreende esses dois artefatos estratégicos, no sentido de diferenciar definições e refletir sobre suas estruturas, bem como suas aplicações nas organizações, o que pode auxiliar na obtenção de vantagem competitiva, tendo em vista que a limitação por informações estratégicas pode impactar negativamente a prática e consequente obtenção de resultados.

O trabalho teve como premissa principal o estudo das diferenças e semelhanças entre o CTC e o CCV. Após o desenvolvimento e apresentação das discussões é possível responder o questionamento: quais as diferenças e semelhanças entre o CTC e o CCV?

Os resultados levaram a percepção de que as principais diferenças encontradas se devem ao fato do CCV compreender desde a concepção do produto até seu descarte, enquanto o CTC inicia no momento da aquisição do produto e termina igualmente com o descarte. Assim, identificou-se uma intersecção pela qual - CTC compõe parte do CCV. O foco tratado pelos dois artefatos também pode ser considerado uma diferença relevante. O CTC é exposto na literatura, geralmente, como uma ferramenta de compra, enquanto o CCV uma ferramenta de gestão de custos mais completa, ou seja, que abrange os custos do "berço ao túmulo". Quanto às semelhanças, pode-se citar que os dois caminham rumo à obtenção de vantagem competitiva por meio da exploração das etapas produtivas ou de consumo, finalizando na etapa do descarte. Também se atenta para a relevância da utilização em conjunto dos dois artefatos. Isso proporciona uma análise mais ampla, tendo em vista a visão e compreensão completa do processo produtivo, com ênfase especial ao período em que o produto é adquirido e consumido. 
Em consonância a essa relevância e ao objetivo do estudo, é provável também que a falta de compreensão das definições dos artefatos estratégicos pelas empresas pode ser custosa, além de induzir a perda de competitividade. Decisões sem base confiável para análise, podem afetar negativamente a lucratividade da empresa, visto que os custos podem superar os preços de venda de produtos/serviços. Por isso, é importante reforçar a necessidade da correta aplicação dos artefatos, partindo do entendimento de suas funções, diferenças e semelhanças.

Por fim, recomenda-se para futuras pesquisas, verificar o nível de utilização prática dos dois artefatos pelas organizações e a forma como vem sendo usados, bem como a percepção dos gestores, controllers e analistas de custos das organizações de diferentes segmentos e portes em relação ao conhecimento existente entre os dois artefatos, visto a importância desse tipo de informação em relação à difusão dos mesmos no ambiente empresarial.

\section{Referências}

AMARAL, J. V; SOUZA, M. F. Diferentes Barreiras à análise do Custo Total para os consumidores. Revista ABCustos-São Leopoldo -Associação Brasileira e Custos. v. 11, n. 2. pp. 28-48, 2016.

BERLINER, C.; BRIMSON, J. Gerenciamento de custos em indústrias avançadas. São Paulo: T. A. Queiroz, 1992.

CARR. L. P.; ITTNER, C. D. Measuring the cost of ownership. Journal of cost management, Fall, p. 42-49, 1992.

DEGRAEVE, Z; LABRO, E.; ROODHOOFT, F. An evaluation of vendor selection models from a total cost of ownership perspective. European Journal of Operational Research, v. 125, n. 1, p. 34-58, 2000.

DIAS FILHO, J. M.; NAKAGAWA, M.; ROCHA, W. A relação entre o custeio do ciclo de vida do produto e a obtenção de vantagem competitiva sustentável: abordagem da gestão estratégica de custos. In: CONGRESSO BRASILEIRO DE CUSTOS, 9, 2002, São Paulo. Anais... São Paulo: 2002.

ELLRAM, L. M. Total cost of ownership: an analysis approach for purchasing. International Journal of Physical Distribution and Logistics Management, v. 25, n. 8, p. 4-23, 1995.

ELLRAM, L. M; SIFERD, P. Total cost of ownership: a key concept in strategic cost management decisions. Journal of Business Logistics, v. 19, n. 1, p. 55-84, 1998.

ELLRAM, L. M.; ZSIDISIN, G. A. Factors that drive purchasing and supply management's use of information technology. IEEE Transactions on Engineering Management, v. 49, n. 3, p. 269-281, 2002.

FERRIN, B. G. PLANK, R. E. Total cost of ownership models: an exploratory study. Journal of Supply Chain Management, v. 38, n. 3, p. 18-29, 2002. 
HANSEN, D. R.; MOWEN, M. M. Gestão de custos: contabilidade e controle. 3. ed. São Paulo: Cengage Learning, 2010.

HEILALA, J.; MONTONEN, J.; HELIN, K. Selecting the right system - assembly system comparison with total cost of ownership methodology. Assembly Automation, v. 27, n. 1, p. 44-54, 2007.

ILG, P; HOEHNE, C; GUENTHER, E. High-performance materials in infrastructure: a review of applied life cycle costing and its drivers - the case of fiber-reinforced composites. Journal od Cleaner Production. v. 112, pp. 926-945, 2016.

LANGFIELD-SMITH, K. Strategic management accounting: how far have we come in 25 years? Accounting, Auditing \& Accountability Journal, v. 21, n. 2, pp. 204-228, 2008.

MENDES, R. C; BARBOSA NETO, J. E.; DIAS, W. O. Custo Total de Propriedade: análise da utilização nas decisões de investimentos em bens de capital. Revista Brasileira de Contabilidade. n. 194. pp. 86-99, 2012

$\mathrm{MIAH}$, J. H; KOH, S. C. L; STONE, D. A hybridised framework combining integrated methods for environmental Life Cycle Assessment and Life Cycle Costing. Journal of Cleaner Production. v. 168, pp. 846-866, 2017.

MICHAELIS. Moderno dicionário da língua portuguesa. Disponível em: http://michaelis.uol.com.br/moderno/portugues/index.php. Acesso em 17 jul, 2015.

MORSSINKHOF, S.; WOUTERS, M.; WARLOP, L. Effects of providing total cost of ownership information on attribute weights in purcashing decisions. Journal of Purchasing \& Supply Management, v. 17. N. 2, p. 132-142, 2011.

NAKAGAWA, M. Gestão estratégica de custos: conceito, sistemas e implementação. São Paulo: Atlas, 1993.

OKANO, K. Life cycle costing - An approach to life cycle cost management: A consideration from historical development. Asia Pacific Management Review. v. 6, n. 3, pp. 317-341, 2001.

PEREZ JUNIOR, J. H.; OLIVEIRA, L. M.; COSTA, R. G. Gestão estratégica de custos, 4 ed. São Paulo: Atlas, 2005.

SACCANI, N; PERONA, M; BACCHETTI, A. The total cost of ownership of durable consumer goods: a conceptual model and an empirical application. Int. J. Econimics Journal. 183, pp. 1-13, 2017

SAKURAI, M. Gerenciamento integrado de custos. 1. ed. São Paulo: Atlas, 1997.

SANTOS, L. J. C; TENÓRIO, J. A. S. Avaliação do ciclo de vida e custeio do ciclo de vida de evaporadores para usinas de açucar. Revista Esc. Minas, Ouro Preto. v. 63, n. 1, pp. 179-184, 2010. 
SCHMIDT, P; SANTOS J. L. D; PINHEIRO, P. R; NUNES, M. M. D. A importância do total cost of ownership no gerenciamento da cadeia de suprimentos. Revista ConTexto-Porto Alegre. v. 13, n. 25, pp. 20-31, 2013.

SHANK, J. K.; GOVINDARAJAN, V. A revolução dos custos: como reinventar e redefinir sua estratégia de custos para vencer em mercados crescentemente competitivos. 2. ed. Rio de Janeiro: Campus, 1997.

SHIELDS, M.D.; YOUNG, S.M. Managing Product Life Cicle Cost. Journal of Cost Management (Fall). pp. 39-51, 1991.

SILVA, A. C. R. Metodologia da pesquisa aplicada à contabilidade. São Paulo: Atlas, 2010.

SILVA, J. O.; ABADE, T.; FEHR, L. C. F. A.; BORINELLI, M.; ROCHA, W. Análise das diferentes abordagens do conceito de custo total para o consumidor: um ensaio teórico. Revista Enfoque Reflexão Contábil, v. 34, n. 3, p. 145-158. 2015.

SLAVOV, T. N. B. Gestão estratégica de custos: uma contribuição para a construção de sua estrutura conceitual. Tese (Doutorado em Contabilidade e Controladoria) Programa de Pós Graduação da Universidade de São Paulo, São Paulo, 2013.

SOUTES, D. O. Custo total de propriedade (TCO): é importante? pra quem?. UNIOESTE, v. 7, n. 13, p. 83-105, 2007.

ZACHARIASSEN, F.; ARLBJORN J.S. Exploring a differentiated approach to total cost of ownership. Industrial Management \& Data Systems, v. 111, n. 3, p. 448-469, 2011.

WERNKE, R. Gestão de custos: uma abordagem prática. 2. ed. São Paulo: Atlas, 2004.

WOUTERS, M.; ANDERSON, J. C.; WYNSTRA, F. The adoption of total cost of ownership for sourcing decisions - a structural equations analysis. Accounting, Organizations and Society, v. 30, n. 2, p. 167-191, 2005.

YAO, F; JIA, Y; MAO, Z. The cost analysis of hydrogen life cycle in China. International Journal of Hydrogen energy, v. 35, pp. 2727-2731, 2010 